\title{
A case of neuromyelitis optica (Devic's disease)
}

\section{SGP Kumuduni ${ }^{1}$, Athula Dissanayaka ${ }^{2}$}

${ }^{1}$ Registrar in Medicine, ${ }^{2}$ Consultant Neurologist, Teaching Hospital, Karapitiya, Galle.

\section{Introduction}

Neuromyelitis optica is an inflammatory disease of the central nervous system in which there are episodes of inflammation and damage to the myelin that almost exclusively affect the optic nerves and spinal cord. It usually causes temporary blindness, occasionally permanent, in one or both eyes. It can also lead to varying degrees of weakness or paralysis in the legs or arms, loss of sensation, and/or bladder and bowel dysfunction from spinal cord damage.

\section{Case report}

A 40 year old previously healthy man was admitted with sudden onset urine retention and weakness of lower limbs extending to upper limbs, associated with sudden loss of vision in both eyes, one week following a febrile illness.

Examination revealed flaccid paraparesis of both lower limbs with a sensory level at thoracic 10 for all sensory modalities. Reflexes were absent in the lower limbs. There was grade 3 weakness of upper limbs. Bladder and bowel functions 
were also affected. Visual acuity was $6 / 12$ in the right eye and $1 / 60$ in the left eye. Both optic discs were pale with bilateral relatively apparent papillary defect.

His CSF was normal. MRI scan of the thoracic spine and brain revealed demyelination. With the clinical and investigation findings the diagnosis of a rare disease neuromyelitis optica was made and treatment started with high dose steroids (methylprednisolone $500 \mathrm{mg}$ daily for 5 days) and plasma exchange. Unfortunately patient did not recover fully although his upper limb weakness has improved.

\section{Discussion}

Neuromyelitis optica often follows a sore throat, common cold or other febrile illness. Either the ocular or the spinal lesion may develop first, separated by days or weeks or both may occur simultaneously. The ocular lesion may be an optic neuritis or a retro bulbar neuritis. The spinal cord lesion which may be heralded by severe pain in the back and limbs lead to usual features of transverse myelitis with paralysis of upper motor neurone type and loss of sensation below the level of the lesion and of sphincter control ${ }^{1}$.

There are two major types of neuromyelitis optica. In the first type, optic neuritis and myelitis episodes tend to come very close together often within days or weeks and there is no recurrence after the initial flurry of symptoms. In the second form, repeated episodes of optic neuritis and myelitis that are separated by months or years occur ${ }^{1}$.The combination of neurological impairments which occur in patients with neuromyelitis optica can also be seen in multiple sclerosis (MS), acute disseminated encephalomyelitis (ADEM), systemic lupus erythematosus (SLE) and Sjögren syndrome.

CSF may show no abnormality or there may be an increase of protein and globulin, an excess of cells, usually mononuclear though occasionally neutrophils, have been described. There is usually an increase in oligoclonal immunoglobulins. Longitudinally extensive transverse myelitis (LETM) is the usual spinal cord finding in the MRI of the neuromyelitis optica ${ }^{2}$. The MRI findings of neuromyelitis optica can to some degree overlap with MS but in general MS has discrete lesions. The MRI of the brain would be initially normal. But later on it may reveal MS like white matter lesions. The morphology and distribution of these lesions tend to differ from that in MS ${ }^{2}$. The defining hallmark of this disease at this time is the identification in the patient's serum of a particular antibody, the NMO - IgG antibody that appears against a particular protein - an aqua protein - that is located in areas that are affected in neuromyelitis optica. It is a reliable biomarker to distinguish neuromyelitis optica from MS. This antibody seems to be present in about 70 percent of patients with neuromyelitis optica and is not found in people with MS or other similar conditions ${ }^{3}$.

There is no cure for neuromyelitis optica, but there are therapies to reduce symptoms, and to prevent relapses. Usually the initial attack of neuromyelitis optica is treated with a combination of a corticosteroid drug (methylprednisolone) to stop the attack, and an immunosuppressive drug (azathioprine) for prevention of subsequent attacks. If frequent relapses occur, some individuals may need to continue a low dose of steroids for longer periods. Plasma exchange is used for people who are unresponsive to corticosteroid therapy. Pain, stiffness, muscle spasms, and bladder/ bowel control problems can be managed with the appropriate medications and therapies. Individuals with major disability will require the combined efforts of occupational therapists, physiotherapists and social services professionals to address their complex rehabilitation needs ${ }^{4}$.

\section{References}

1. John Walton. Disseminated myelitis with optic neuritis In Brain's diseases of the nervous system, $9^{\text {th }}$ edition, 306- 307

2. DAS Compston. Demyelinating disorders of the central nervous system. In: David A.Warrell,Timothy M.Cox,John D. Firth,Edward J.Benz Jr. Oxford Textbook of Medicine, $4^{\text {th }}$ edition, Vol 3, 2003: 1158. 


\section{Case Reports}

3. Lennon VA, Wingerchuk DM, Kryzer TJ. A serum autoantibody marker of neuromyelitis optica: distinction from multiple sclerosis. Lancet 2004 Dec11; 364(9451): 2106-12.

4. Neuromyelitis optica information page. The National Institute of Neurological Disorders and Stroke(NINDS), $5^{\text {th }}$ March 2007 update. 